\title{
James Webb Space Telescope (JWST) Optical Telescope Element (OTE) Development Status
}

\author{
Lee D. Feinberg, JWST Optical Telescope Element (OTE) Manager \\ Code 443, NASA Goddard Space Flight Center \\ Greenbelt, MD 20771 \\ Lee.D.Feinberg@Nasa.Gov
}

\begin{abstract}
The James Webb Space Telescope (JWST) Optical Telescope Element (OTE) is a segmented, cryogenic telescope scheduled for launch in 2011. In September of 2002, NASA selected prime contractor Northrop Grumman Space Technology (NGST) to build the observatory including management of the OTE. NGST is teamed with subcontractors Ball Aerospace, Alliant Techsystems (ATK), and Kodak. The team has completed several significant design, technology, architecture definition, and manufacturing milestones in the past year that are summarized in this paper.
\end{abstract}

\section{INTRODUCTION}

NASA plans to launch the James Webb Space Telescope into space in less than a decade. The observatory will be launched in a stowed configuration on an expendable rocket and will deploy to its full 6.6 meter diameter on its way to reaching its final destination at the L2 Lagrange point. This location provides an optimal location for passively cooling the telescope to approximately 30 Kelvin using a deployed sunshade. The cryogenic temperature of the telescope was chosen to allow it to operate in the near- and mid-infrared (.7um-28um). These wavelengths are critical to achieve the major science objectives of the telescope because of the fact that starlight from the earliest light in the universe is redshifted into these wavelengths. In addition, the telescope has a collecting aperture greater then 25 square meters in order to achieve sensitivities required to see first light objects.

Due to the mass and volume requirements of the rocket, the telescope will be deployed and have a primary mirror comprised of 18 light-weighted hexagonal-shaped segmented mirrors that will be aligned in space. The mirror technology was recognized as a critical technological challenge early in the program and, through an early start and significant investments by NASA and the prime contractor team, the technology readiness of the lightweight cryogenic mirrors is at a level commensurate with programs much further along in their design cycle. In addition, NASA and the prime contractor team made several early investments in the wavefront sensing and control technology which have allowed the team to demonstrate key aspects of the technology on various testbeds and through modeling.

In addition to the technology developments, a significant amount of other work on the JWST telescope has also started. The OTE System Requirements Review (SRR) was successfully completed in March. Many of the key interface trades have been completed and interface control documents have been baselined. Designs for the primary mirror segments are far along and designs and subsystem requirements for the rest of the system have begun. The primary mirror fabrication has started and most of the key subcontractors are already under contract including mirror manufacturing subcontractors. In addition, the team has finalized facility plans for assembly and testing of the telescope.

\section{ARCHITECTURF}

The first trade study occurred during the program replan and resulted in the decision to baseline the primary mirror collecting area at $25 \mathrm{~m} 2$ with six degree of freedom primary mirror segment articulation. The $25 \mathrm{~m} 2$ was chosen because it met the level 2 requirements and provided mirror schedule risk reduction. The decision to baseline the $25 \mathrm{~m} 2$ aperture size was followed by a segmentation trade study by the prime contractor that selected an 18-segment architecture. The segmentation trade was conducted for both mirror technologies being carried at the time and 18 segments was chosen after looking at many key segmentation issues including integration and testing, wavefront sensing and control, schedule, cryogenic testing, and fabrication issues.

At about the same time as the segmentation trade, a team of optical designers was hard at work determining the prescription of the telescope. A key consideration of this design effort was the F-number of the telescope. The team agreed to baseline an F/20 design that did not add any additional risk to the telescope (e.g., the primary mirror) but that satisfied the desires of the science instruments. 
The team spent several months optimizing the design, including laying out the instrument field-of-views and optimizing the alignment sensitivities of the aft optics and pupils.

The final primary mirror architecture trade decision was the choice of the primary mirror material. The trade was significantly aided by the results of the Advanced Mirror System Demonstrator (AMSD) program, an aggressive mirror technology effort that NASA embarked on early in Phase A. In fact, the trade was timed to make just-in-time use of the cryogenic results of both mirror material options. These cryogenic results proved extremely important in the final selection of Beryllium over glass. The cryogenic results highlighted how the Beryllium mirror's wavefront stability over cryogenic operating temperatures and how it's high thermal conductivity proved to be extremely valuable for the JWST application. However, the trade study also included programmatic considerations such as schedule and facilities where glass had advantages. In the end, a panel of 18 experts assembled and co-chaired by the NGST Telescope Manager and this author recommended the Beryllium option for technical reasons. This recommendation then became the basis for NGST selecting Beryllium as the material for JWST and for NASA approving this decision. Additional details on the primary mirror material recommendation can be found in an accompanying paper by Stahl.

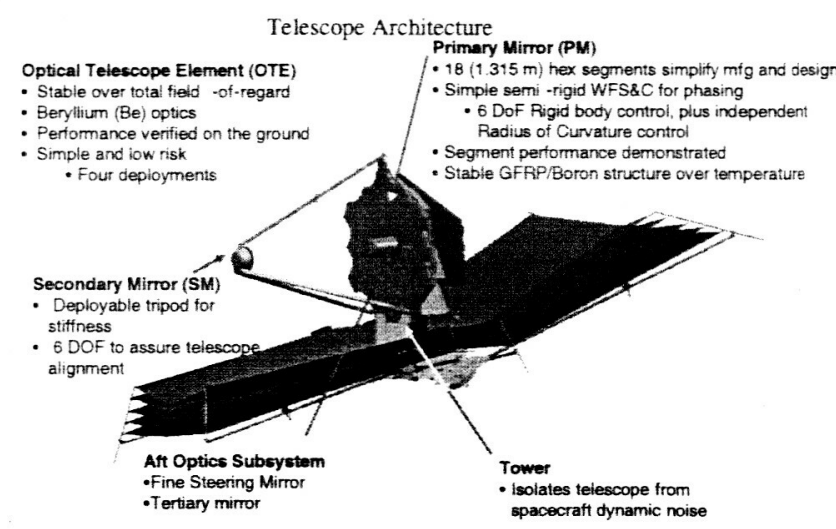

Figure 1 Telescope Architecture

The overall JWST telescope architecture is shown in Figure 1. The telescope is a three-mirror anastigmat that includes a fine-steering mirror. The telescope is an $F / 20$ design and the primary mirror and secondary mirror are deployed in space using mechanisms. The primary mirror is deployed through the use of two wings of three mirror segments each. The secondary mirror requires a more complicated sequence in which it is deployed into capture range. Further details on this architecture can be found on the JWST web page (WWW.JWST. Nasa.gov).

\section{TeChNology Status}

There two major technology challenges for the JWST telescope are the lightweight mirror technology and the wavefront sensing and control algorithms and hardware. The mirror technology is challenging because of the need for low areal density mirrors that can operate at cryogenic temperatures. As a result of AMSD, this technology is fairly far along. A picture of the Beryllium AMSD mirror segment is shown in Figure 2. The AMSD mirror shown met nearly all JWST specifications and was very close in size to the actual JWST mirror segments. The mirrors were extremely repeatable and stable at cryogenic temperatures. At this point in time, most of the remaining technology efforts are focused on optimizing the production of 18 of these mirrors and on a demonstration of an Engineering Development Unit (EDU) that is built exactly to flight specifications.

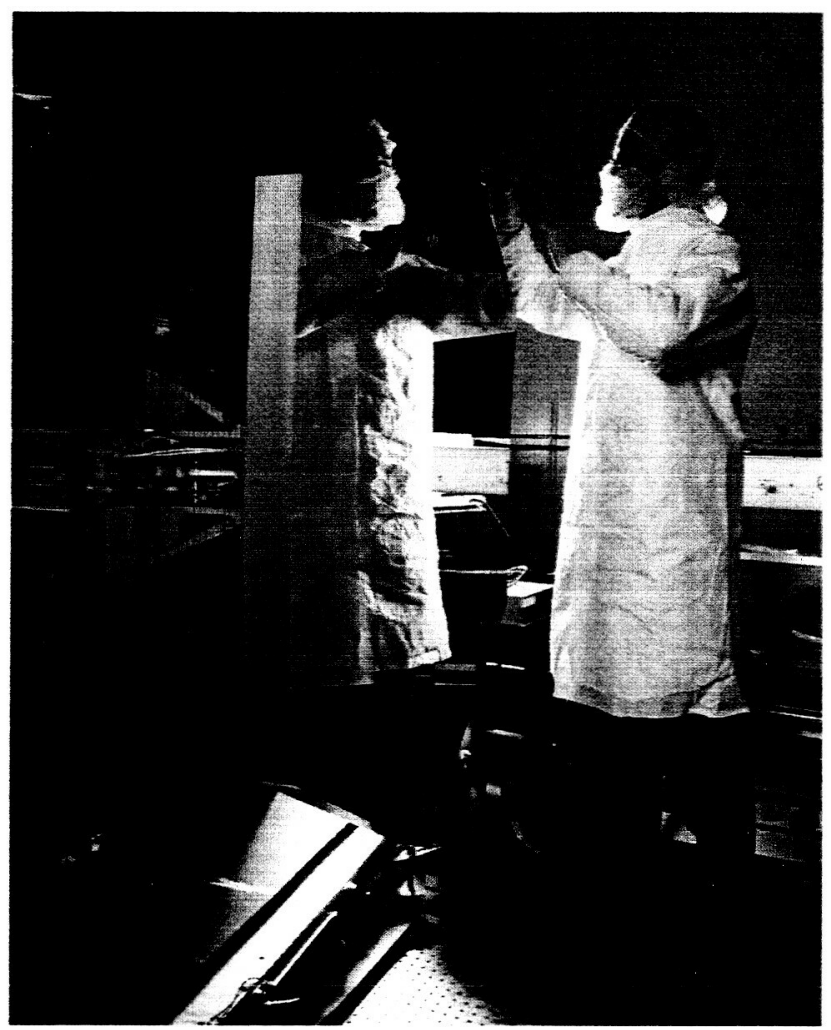

Figure 2 AMSD Beryllium Mirror

The only critical milestone left in achieving Technology Readiness Level -6 , a key need for entering program 
phase $\mathrm{C}$, is the completion of the flight-like vibration test of the EDU mirror

The second key technological challenge, the wavefront sensing and control system, has been demonstrated piecemeal using the Wavefront Control Testbed (WCT) at GSFC and testbeds at Ball Aerospace. Pictures of these testbeds and results from an experiment at Keck are shown in Figure 3. However, these testbeds had a limited number of mirror segments ranging from three to six. Thus, Ball Aerospace is building an 18-segment testbed that is a scaled model of the telescope and which will demonstrate the algorithms to be used on JWST. The wavefront sensing and control system utilizes phase retrieval algorithms that were demonstrated during the spherical aberration prescription retrieval on HST. The system also makes use of grisms for coarse aligning the mirror segments before phase retrieval is employed. The main instrument for JWST, the Near Infrared Camera (NIRCAM), is used as the sensor for wavefront sensing and control.

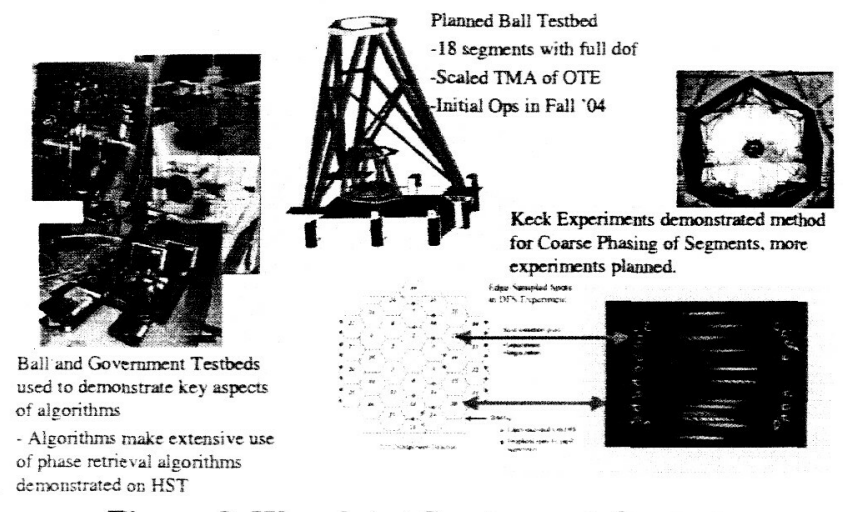

Figure 3 Wavefront Sensing and Control

\section{DeVElopment Status}

The critical path for the JWST mission is the primary mirror of the telescope so early emphasis has been on the design and fabrication of the mirror segments. This work started with a requirements review of the primary mirror segment and design reviews of the mirror substrate and full mirror assembly. Soon after, the manufacturing plans were reviewed at the Mirror Manufacturing Readiness Review (MRR). The mirror design drawings were used to finalize contracts with the mirror subcontractors and are being used in the development of the primary mirror engineering design unit (EDU).

The EDU, shown in Figure 4, is already well into development. The mirror blank was completed at Brush Wellman and the mirror is now being machined at Axsys prior to being sent to SSG/Tinsley for polishing. In addition, the first two flight mirror segment blanks, shown in the right side of Figure 4, are fairly far along at Brush Wellmen. While this work has been going on, facilitization has been ongoing at all of the subcontractors in a rolling wave to support the entire mirror production effort. To date the mirror manufacturing has stayed on schedule through the hard work and dedication of the team as well as experience from the AMSD program.

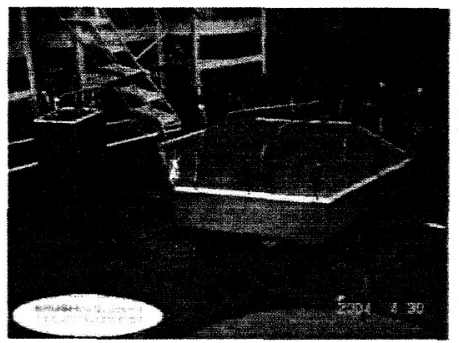

Flight blanks 1 and 2 ready for HIPPING at Brush-Wellman

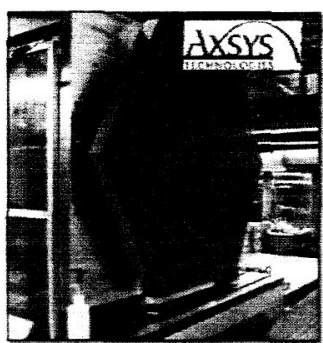

Primary Mirror Segment EDU being Machined at Axsys Technologies

\section{Figure 4 Mirror Fabrication}

The rest of the telescope has also been moving at a fast pace. The System Requirements Review for the telescope was completed in March. The secondary mirror test metrology approach is far along and the backplane design effort and material characterization has begun. The NGST team held an Initial Baseline Review (IBR) at each subcontractor and then for the full program with NASA establishing the cost and schedule program baseline.

Finalization of the wavefront sensing and control architecture has also progressed along with the verification approach. The government team has been independently modeling both the wavefront sensing and control algorithms and the integration and testing architecture and issues raised by the team have been dealt with through design evolution and through risk management. In fact, the prime contractor and NASA team have worked closely on risk management across the entire telescope and this has resulted in risk mitigation plans for each of the critical risks that have been identified.

\section{Conclusion}

Overall, the telescope development is proceeding on schedule. Critical trades have been completed, technologies continue to progress, system requirements have been baselined, initial designs have been completed for the primary mirror segments, and manufacturing of the primary mirror has begun. While new challenges continue 
to arise, the prime contractor and NASA team have worked closely on continuous risk management and risk mitigation plans have been developed for each of the critical risks that have been identified.

\section{CREDITS}

The author would like to thank Scott Texter, the NGST Telescope Manager, and Charlie Atkinson, the NGST Deputy Telescope Manager, for providing inputs to this paper and for their overall stewardship of the telescope at the prime contractor. The author would also like to thank Ritva Keski-Kuha, Lester Cohen, Marcel Bluth, Dave Redding, Dave Van Buren, Phil Stahl, John Hraba, Kevin Russell and the rest of the government team for their work in the technology development and government team oversight of the telescope.

\section{BIOGRAPHY}

Lee Feinberg is the NASA Optical Telescope Element Manager for the JWST telescope. In his previous position

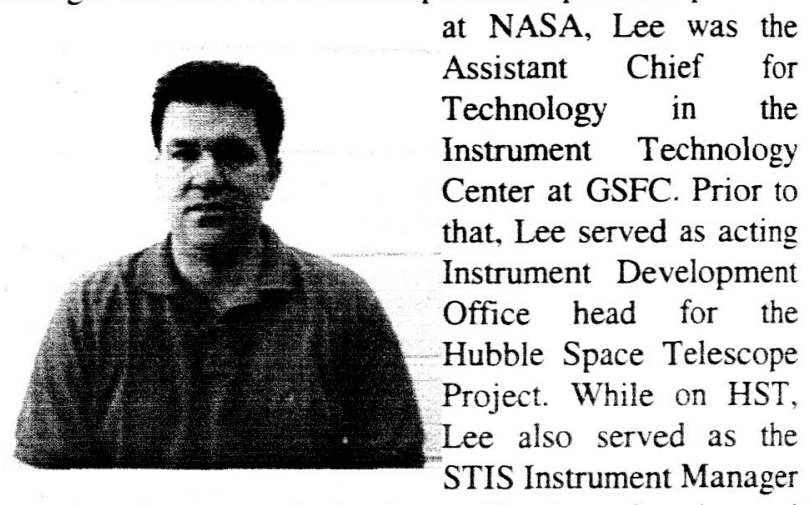
and played a key role in the verification of optics and testing of COSTAR and WFPC-2. Lee also led the Conceptual Study Team for the HST Wide Field Camera3. Before coming to NASA, Lee worked at the University of Rochester's Laboratory for Laser Energetics, at Booz, Allen and Hamilton, and at Ford Aerospace. Lee has a BS in Optics from the University of Rochester and a MS in Applied Physics from The Johns Hopkins University. 

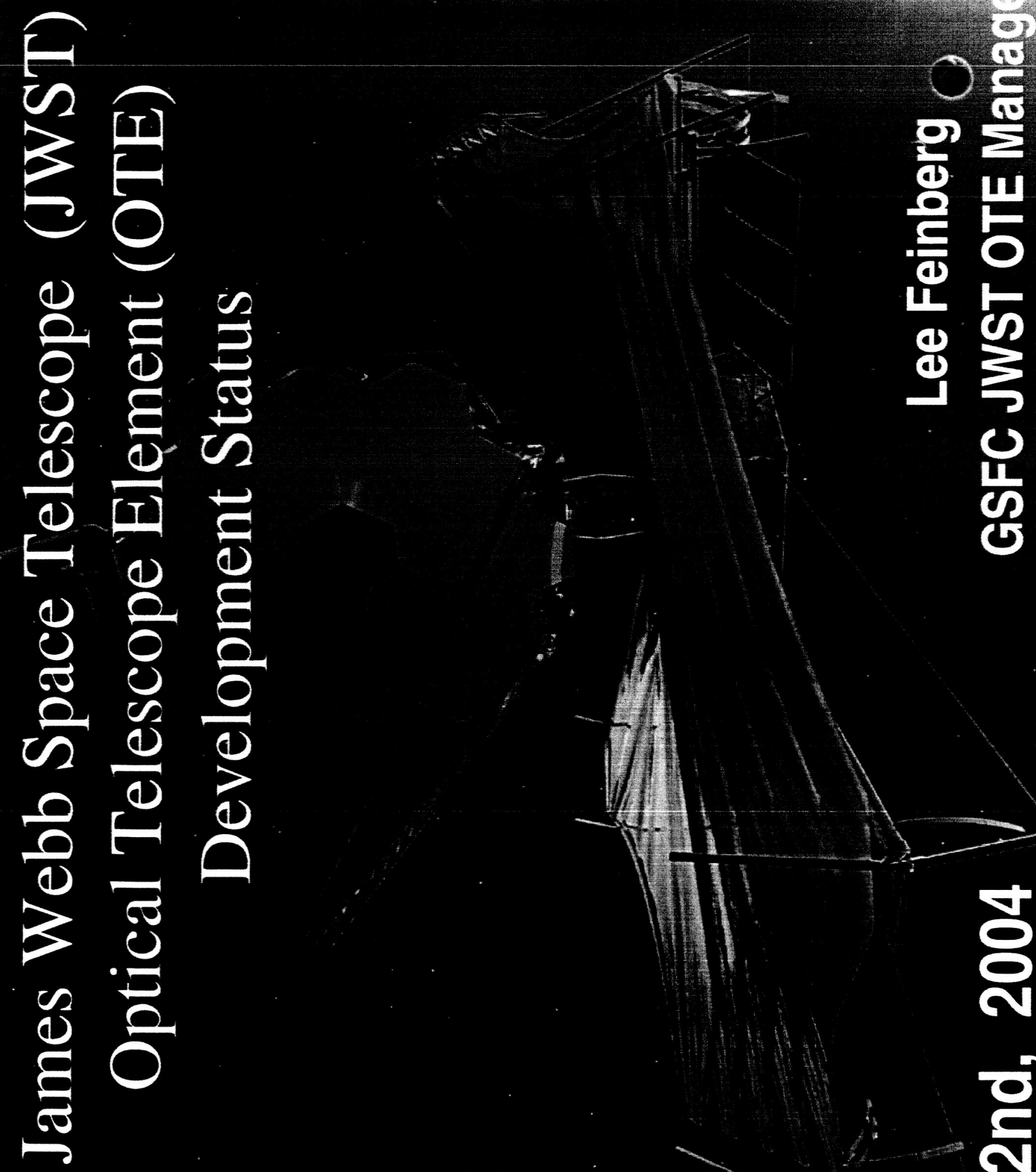

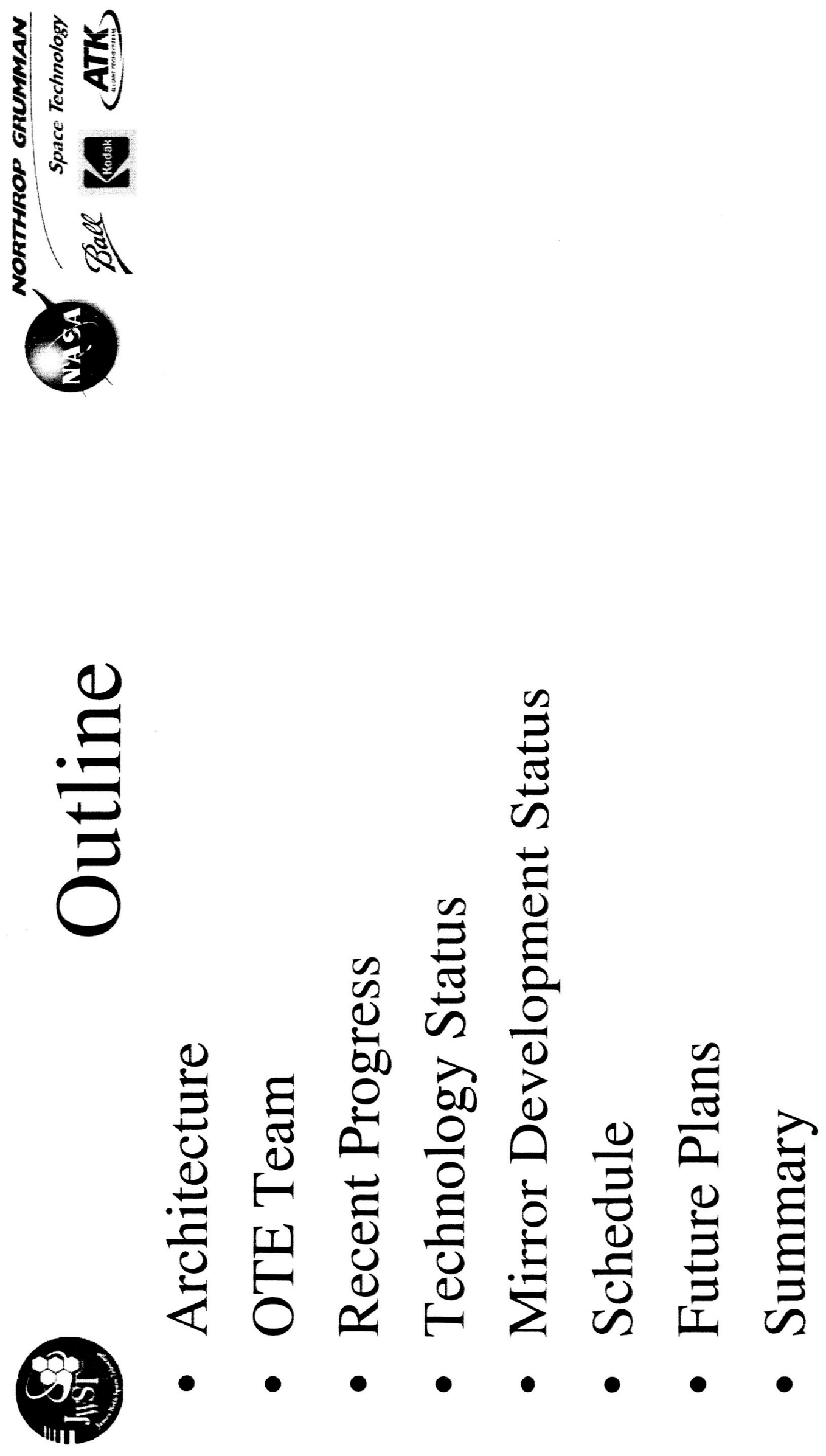


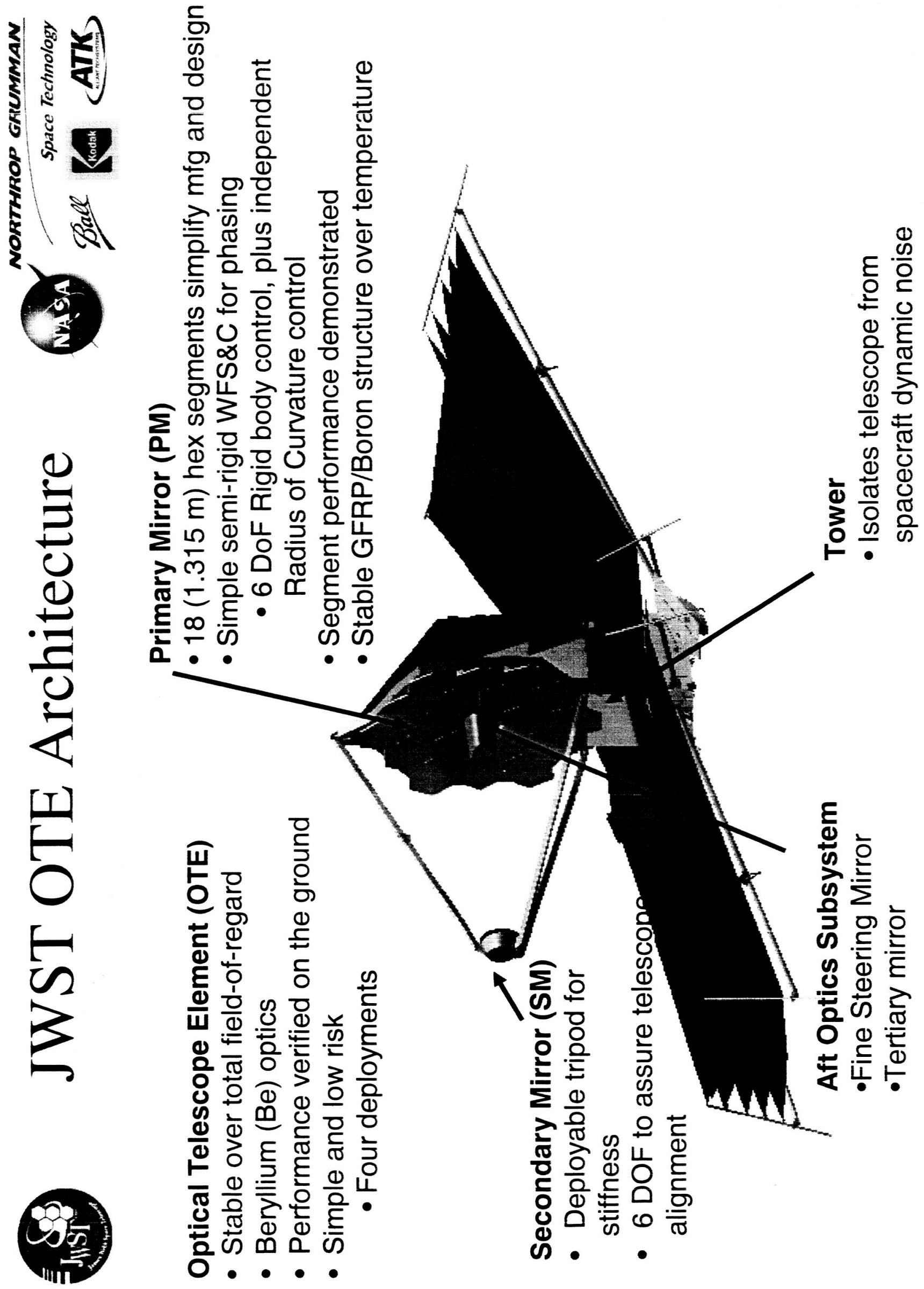



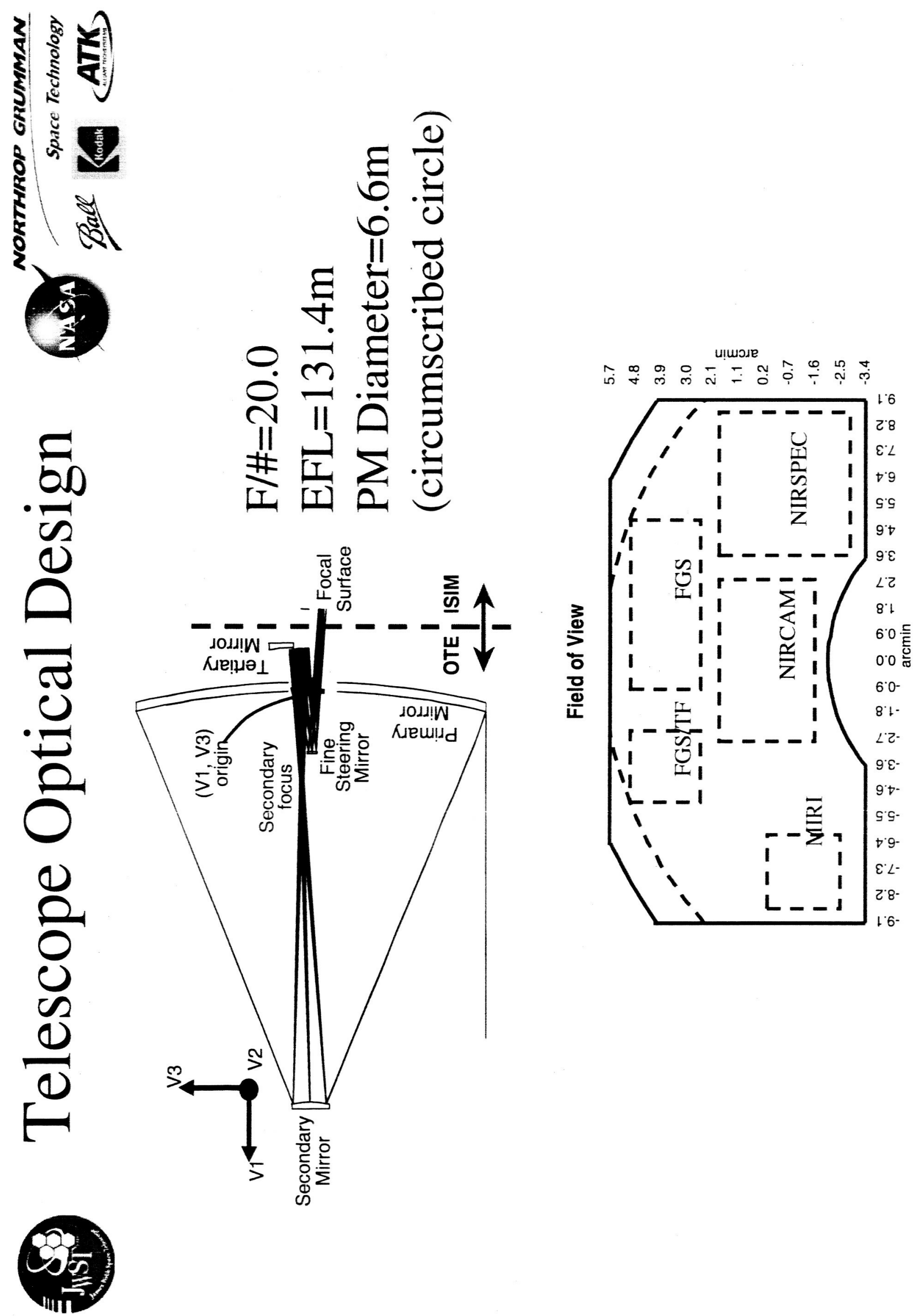

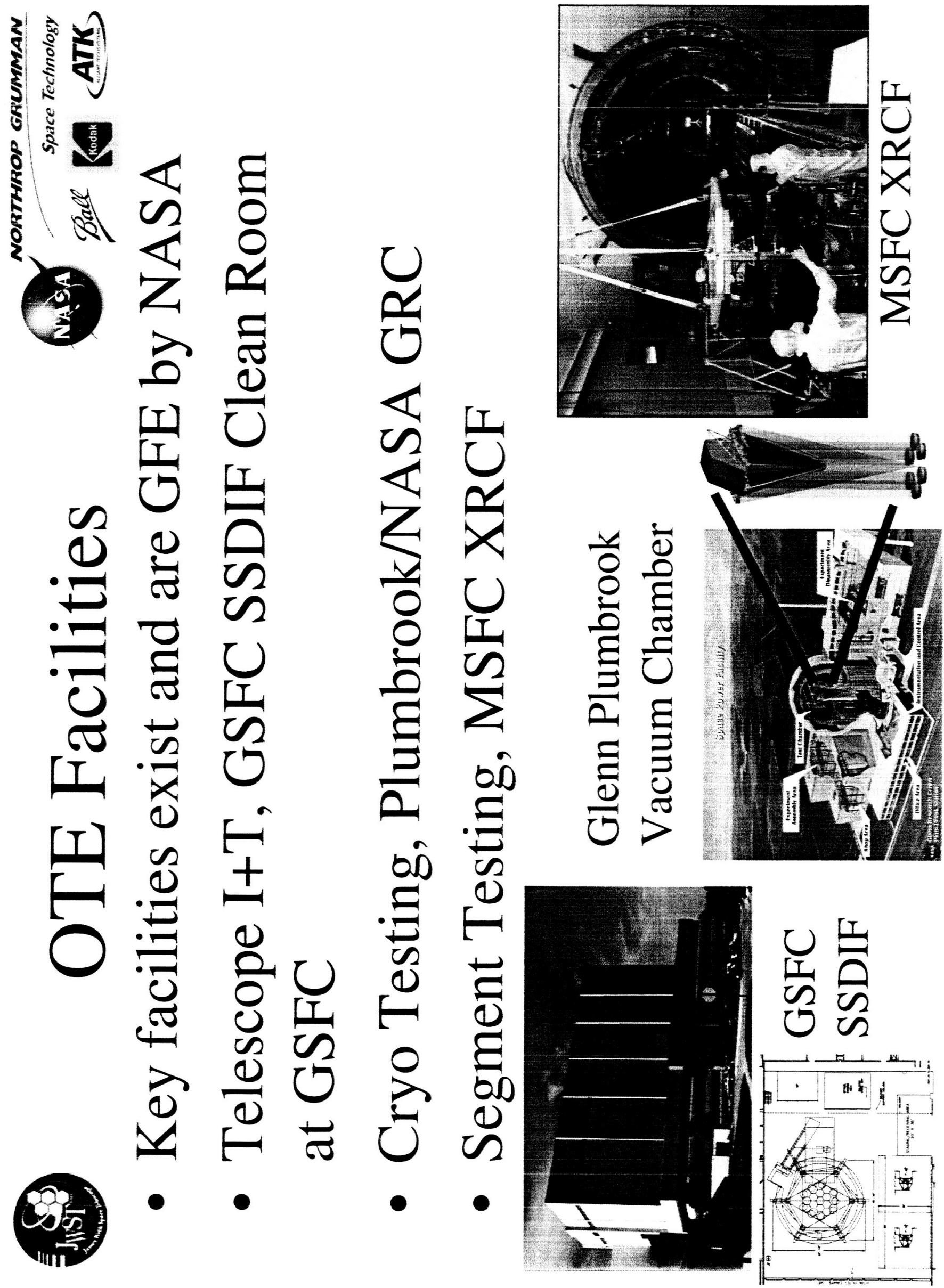


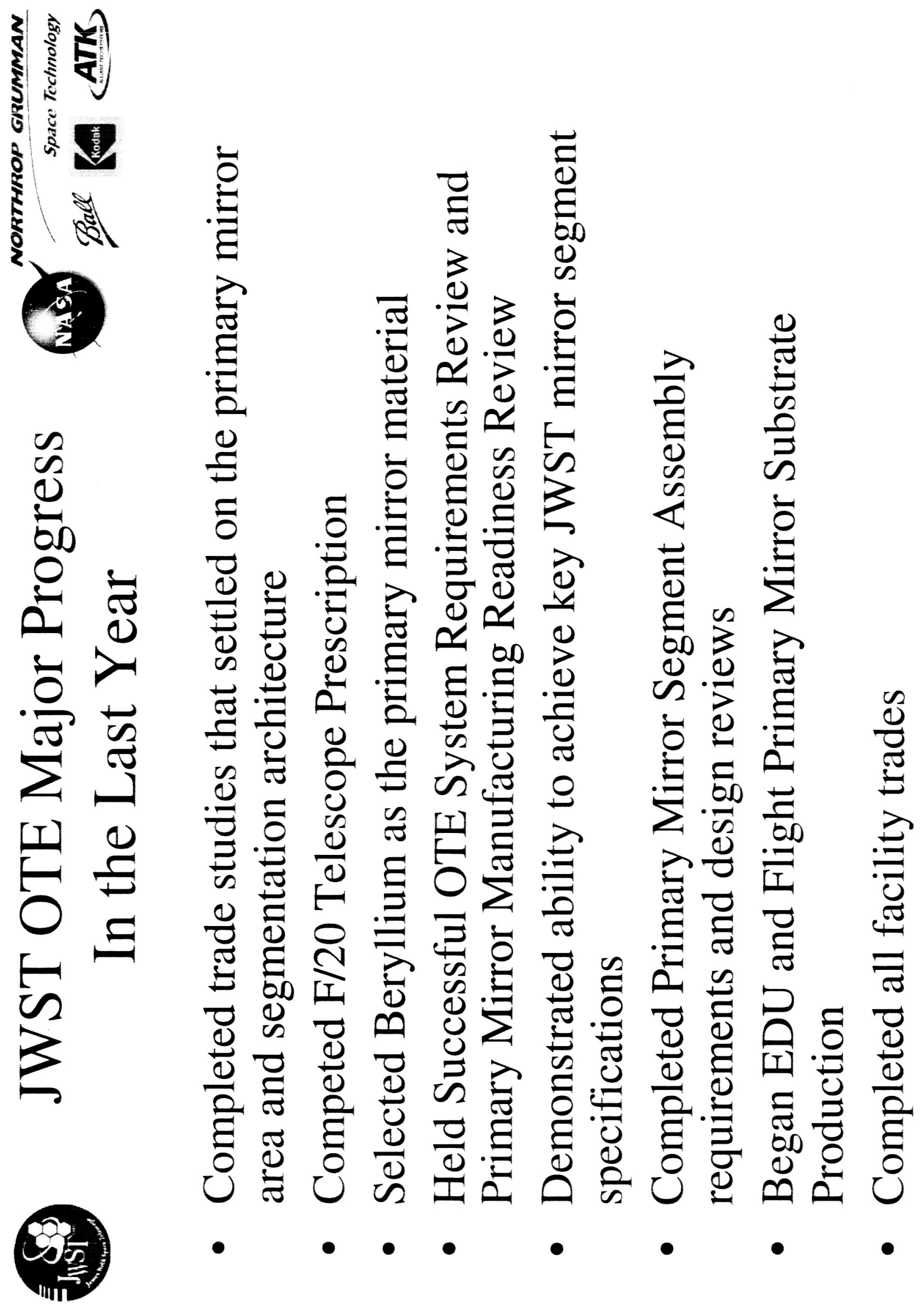




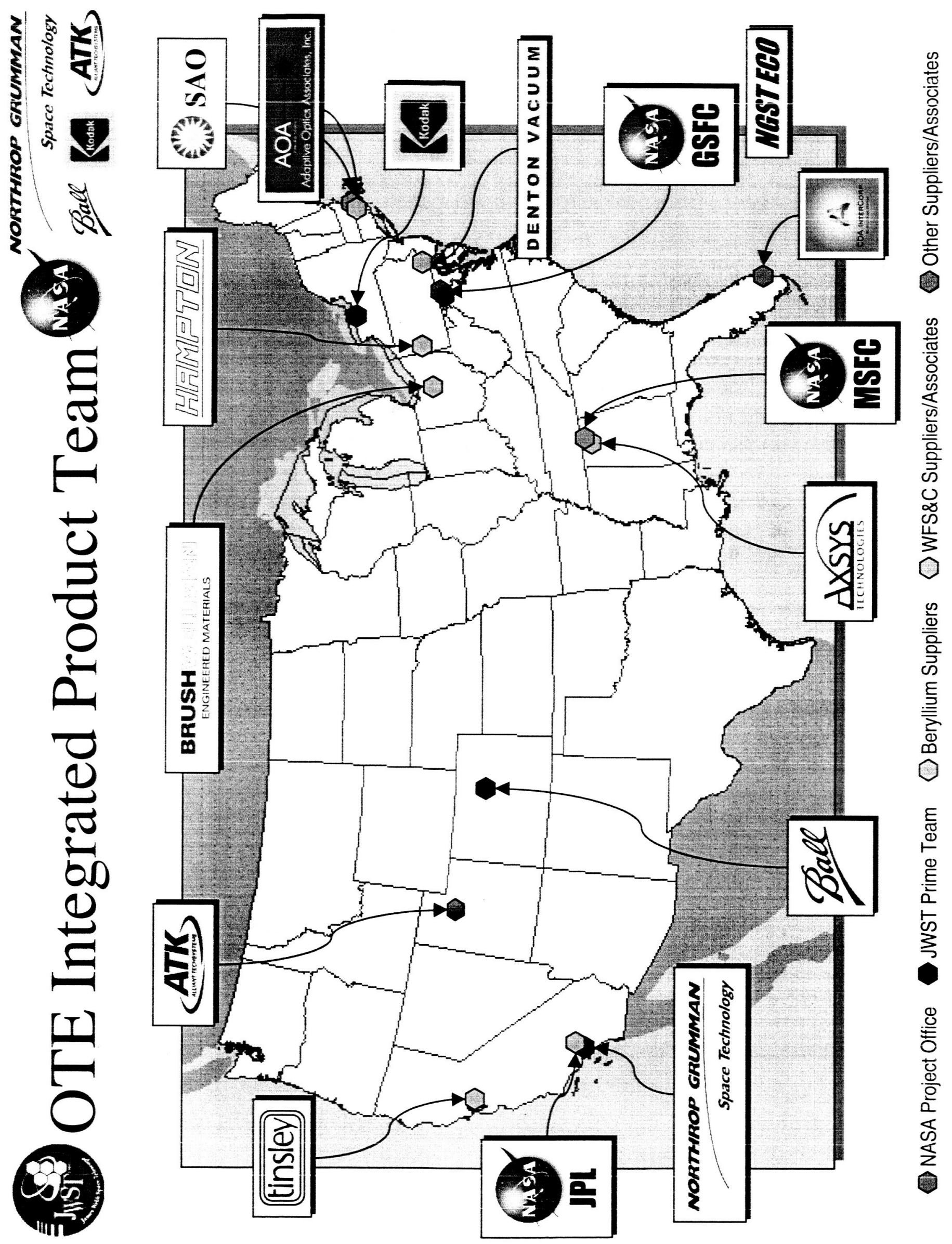




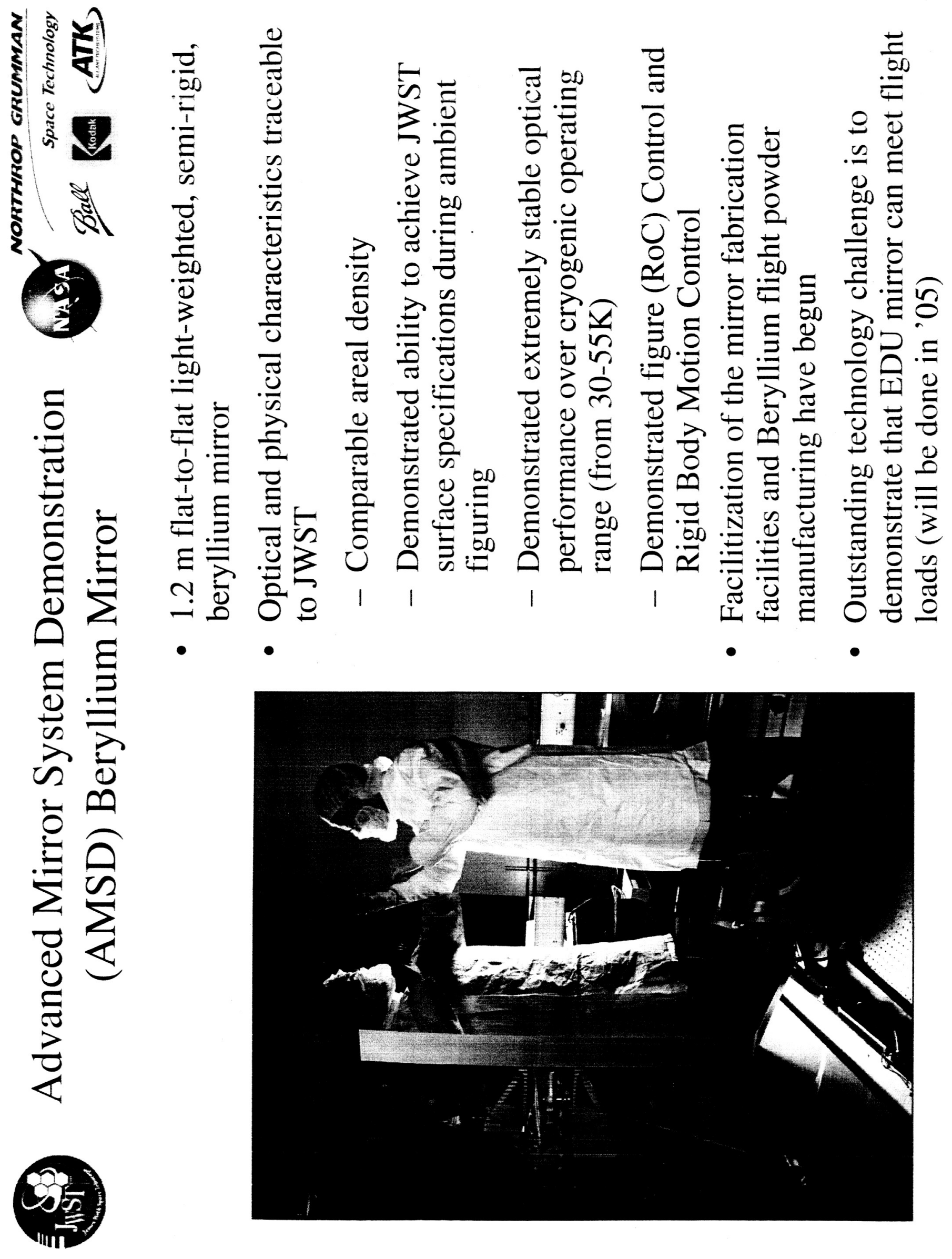



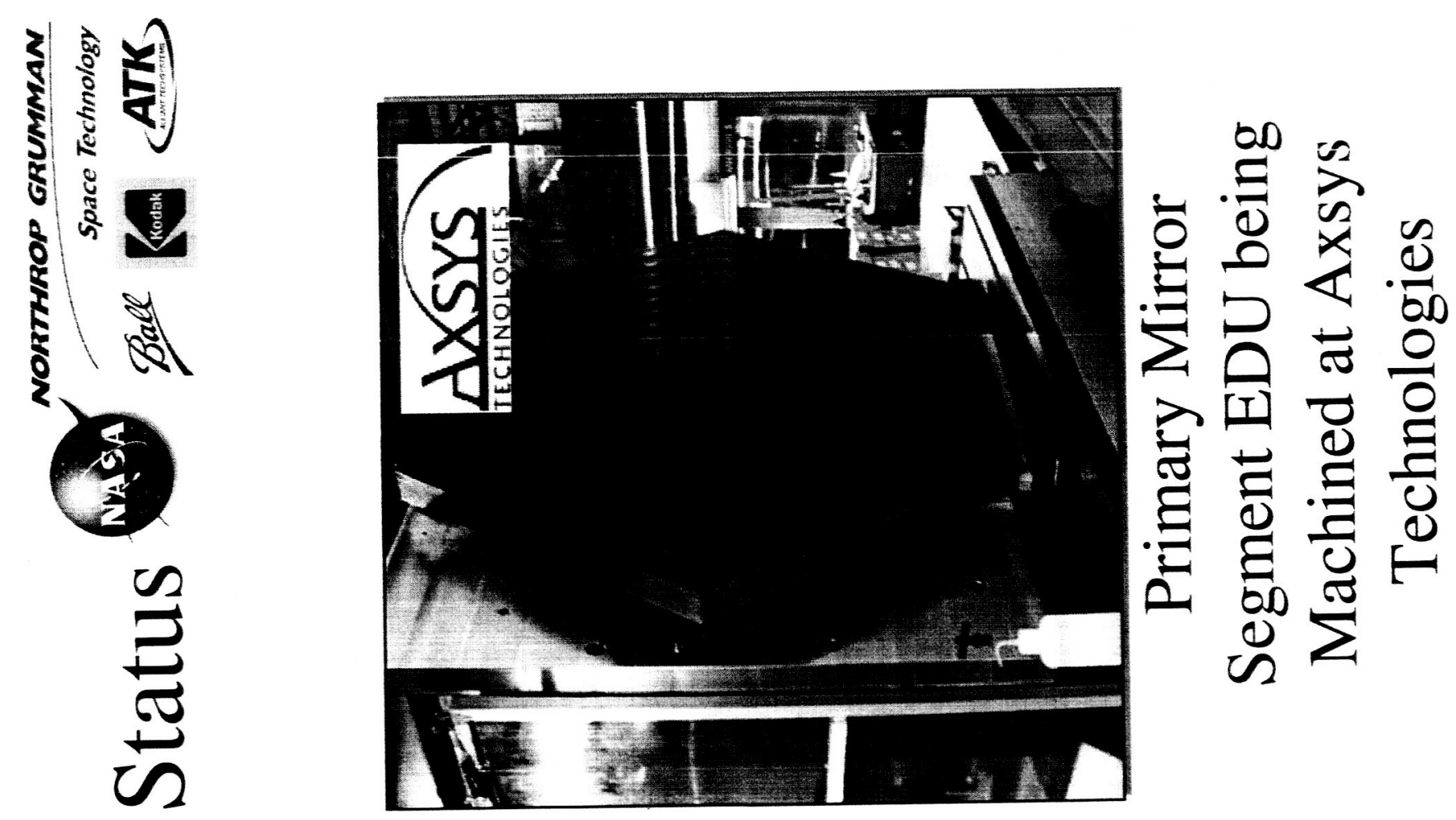

of
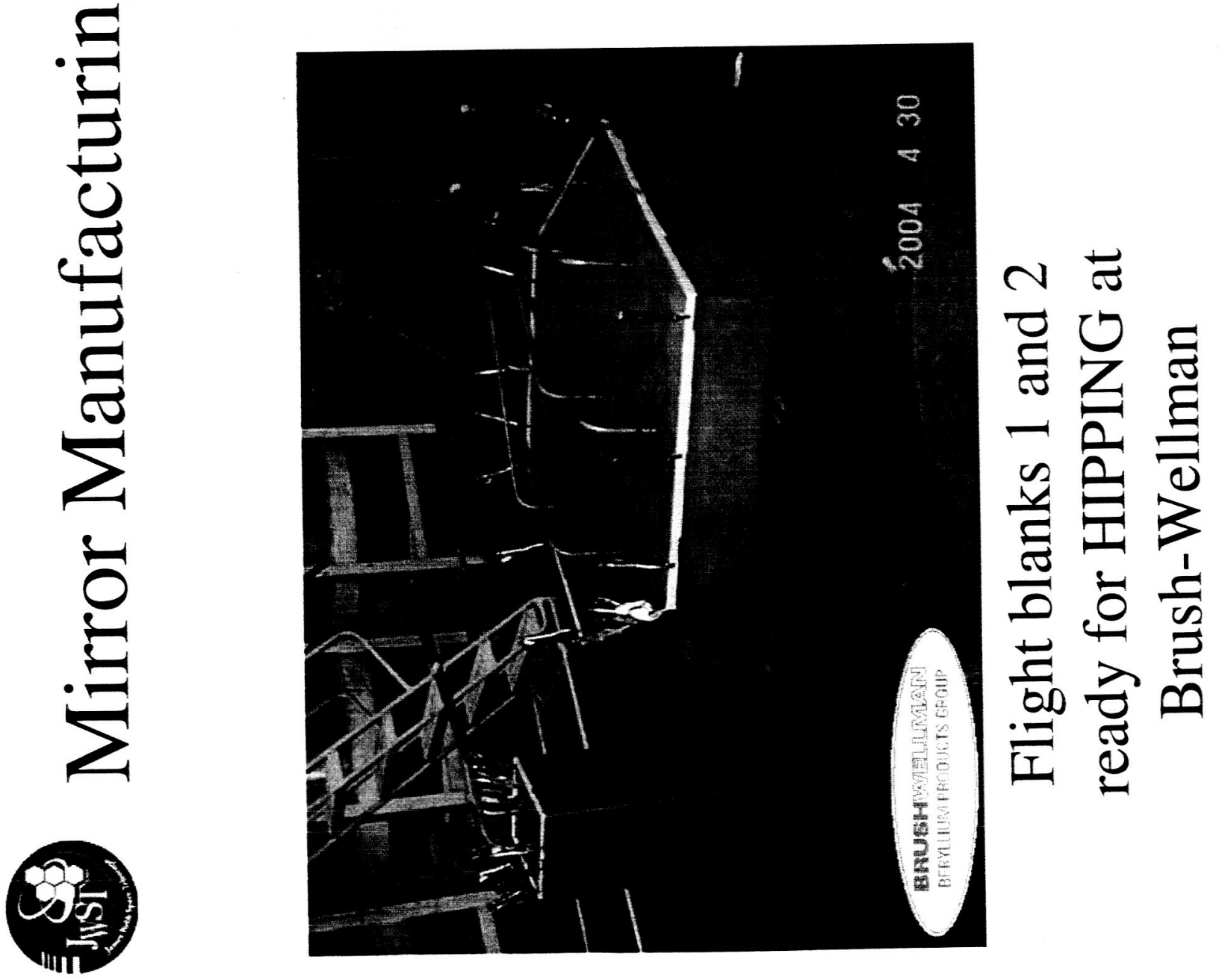

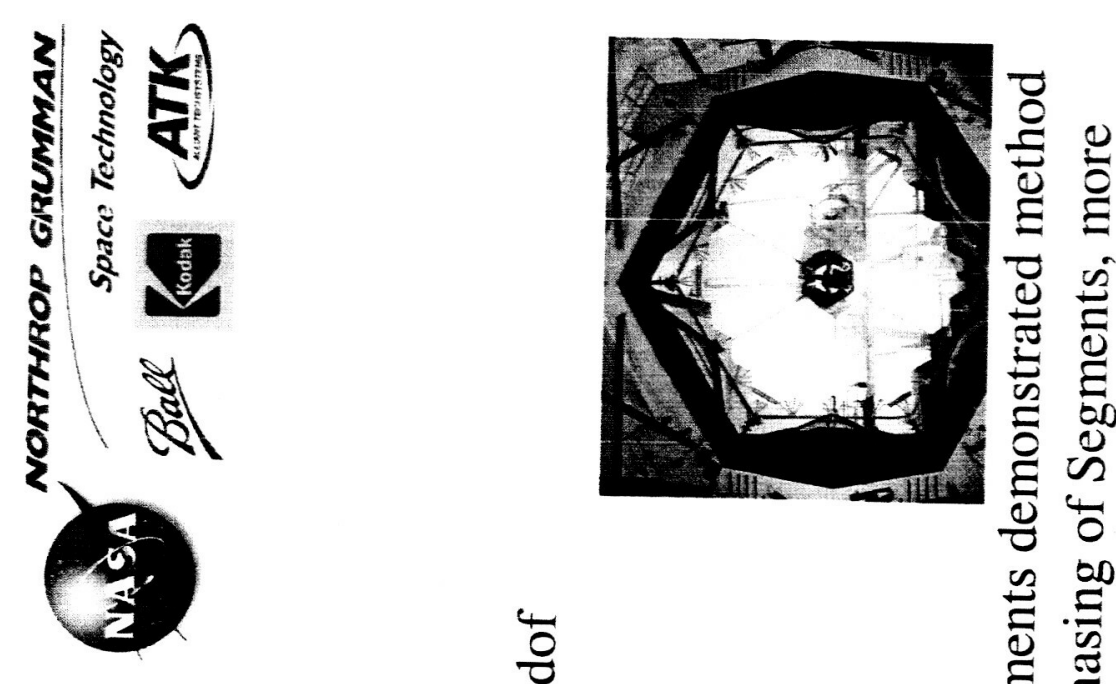

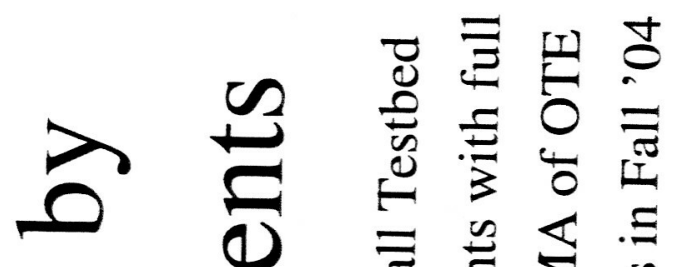
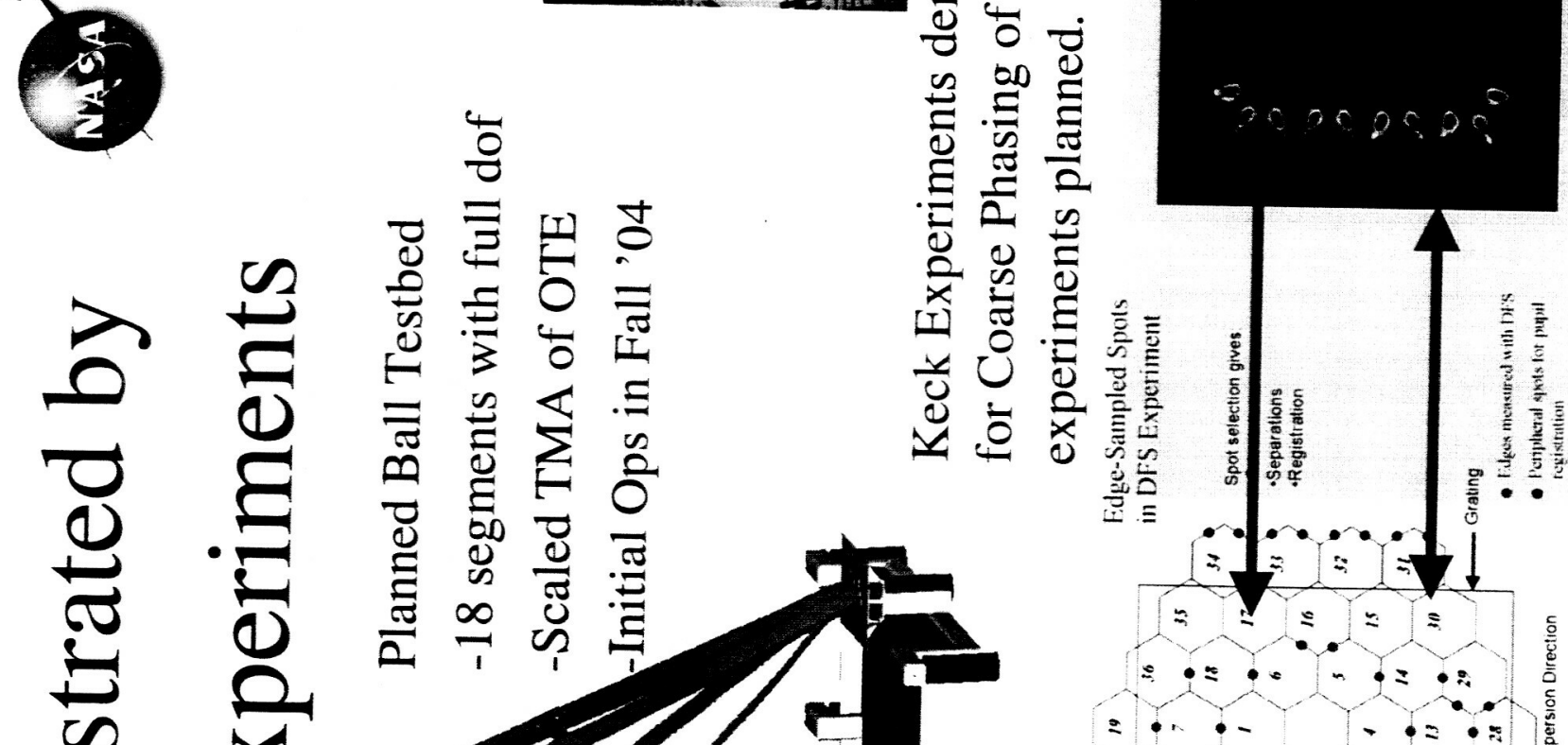


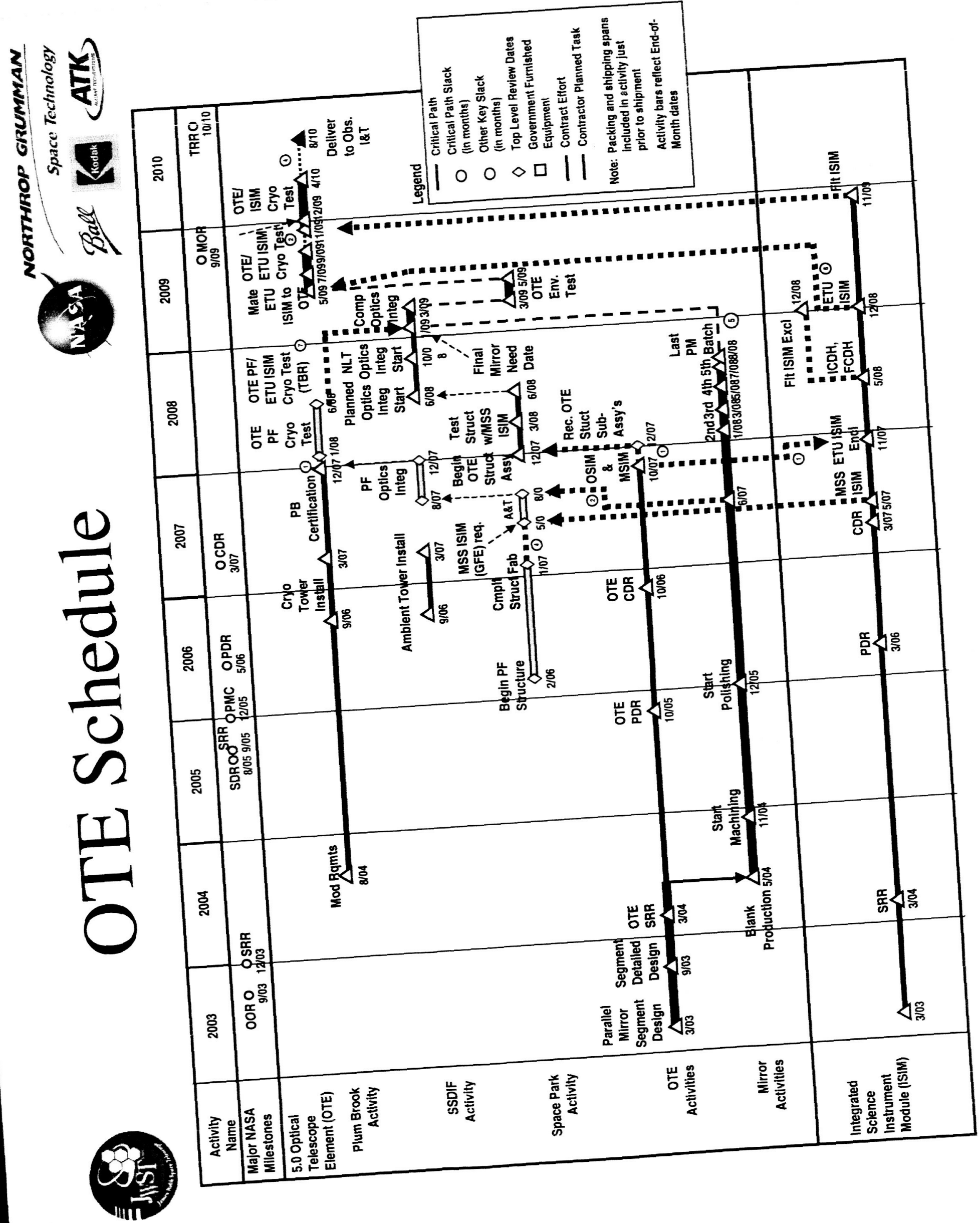




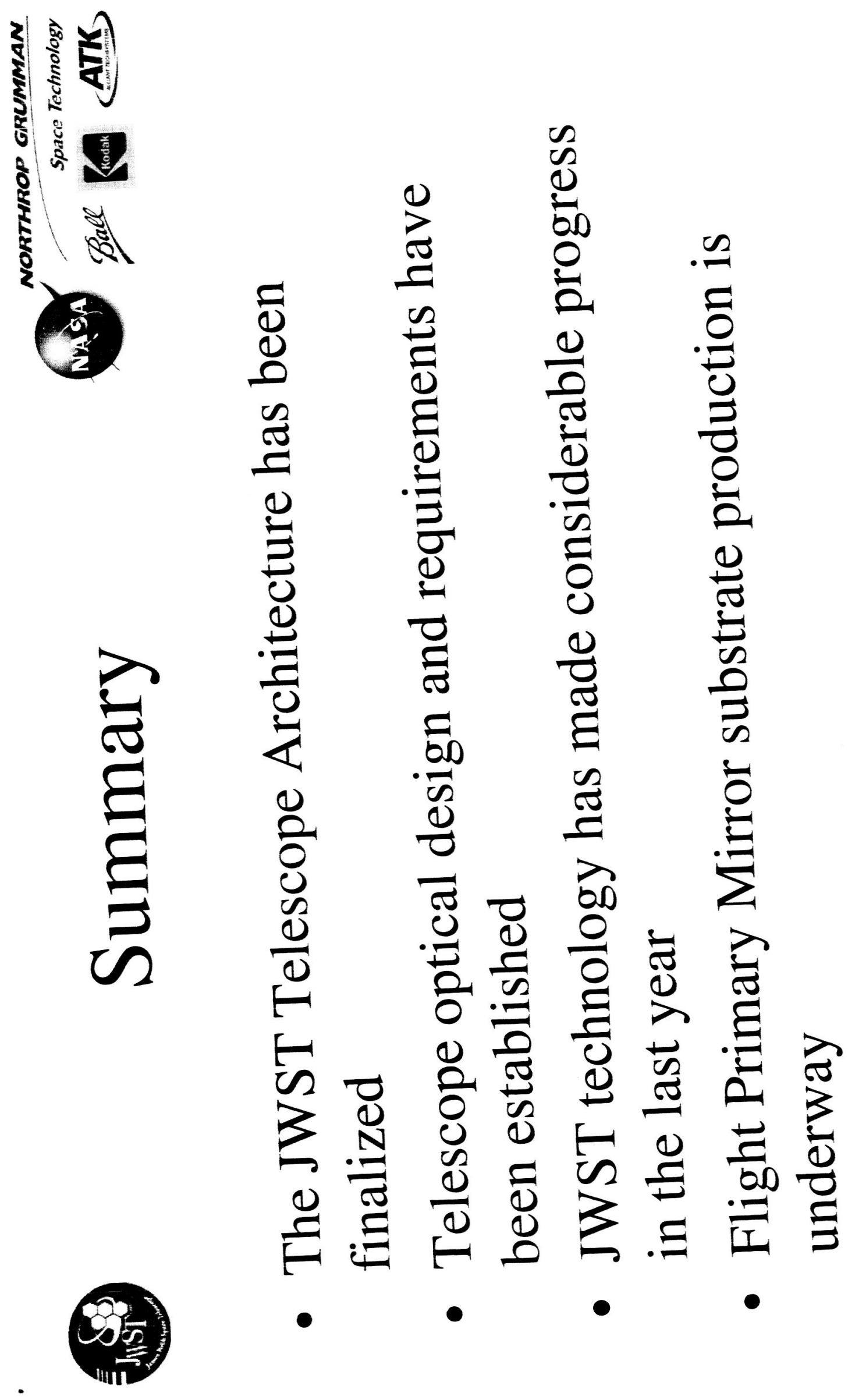

